\title{
Some identities of Barnes-type special polynomials
}

\section{Dongkyu Lim and Younghae Do*}

\section{"Correspondence: yhdo@knu.ac.kr Department of Mathematics, Kyungpook National University, Daegu, 702-701, South Korea}

\begin{abstract}
In this paper, we consider Barnes-type special polynomials and give some identities of their polynomials which are derived from the bosonic $p$-adic integral or the fermionic $p$-adic integral on $\mathbb{Z}_{p}$.

MSC: $11 \mathrm{~B} 68 ; 11540$

Keywords: Barnes-type Bernoulli polynomial; higher-order Euler polynomial; multivariate $p$-adic fermionic integral; Barnes-type Euler polynomial
\end{abstract}

\section{Introduction}

As is known, the Bernoulli polynomials of order $r$ are defined by the generating function to be

$$
\left(\frac{t}{e^{t}-1}\right)^{r} e^{x t}=\sum_{n=0}^{\infty} B_{n}^{(r)}(x) \frac{t^{n}}{n !} \quad(\text { see }[1-29]) .
$$

When $x=0, B_{n}^{(r)}=B_{n}^{(r)}(0)$ are called the Bernoulli numbers of order $r$.

For $a_{1}, a_{2}, \ldots, a_{r} \neq 0 \in \mathbb{C}_{p}$, the Barnes-Bernoulli polynomials are defined by the generating function to be

$$
\prod_{i=1}^{r}\left(\frac{t}{e^{a_{i} t}-1}\right) e^{x t}=\sum_{n=0}^{\infty} B_{n}\left(x \mid a_{1}, a_{2}, \ldots, a_{r}\right) \frac{t^{n}}{n !} .
$$

When $x=0, B_{n}\left(0 \mid a_{1}, a_{2}, \ldots, a_{r}\right)=B_{n}\left(a_{1}, a_{2}, \ldots, a_{r}\right)$ are called Barnes Bernoulli numbers (see [14-33]).

Let $p$ be a fixed odd prime number. Throughout this paper, $\mathbb{Z}_{p}, \mathbb{Q}_{p}$ and $\mathbb{C}_{p}$ will denote the ring of $p$-adic integers, the field of $p$-adic numbers and the completion of algebraic closure of $\mathbb{Q}_{p}$, respectively. The $p$-adic norm is defined as $|p|_{p}=1 / p$. Let $U D\left(\mathbb{Z}_{p}\right)$ be the space of uniformly differentiable function on $\mathbb{Z}_{p}$. For $f \in U D\left(\mathbb{Z}_{p}\right)$, the bosonic $p$-adic integral on $\mathbb{Z}_{p}$ is defined by

$$
\begin{aligned}
I_{0}(f) & =\int_{\mathbb{Z}_{p}} f(x) d \mu_{0}(x)=\lim _{N \rightarrow \infty} \sum_{x=0}^{p^{N}-1} f(x) \mu_{0}\left(x+p^{N} \mathbb{Z}_{p}\right) \\
& =\lim _{N \rightarrow \infty} \frac{1}{p^{N}} \sum_{x=0}^{p^{N}-1} f(x) \quad(\text { see }[16,21]) .
\end{aligned}
$$

\section{Springer}

(0) 2015 Lim and Do; licensee Springer. This is an Open Access article distributed under the terms of the Creative Commons Attribution License (http://creativecommons.org/licenses/by/4.0), which permits unrestricted use, distribution, and reproduction in any medium, provided the original work is properly credited. 
From (2), we have

$$
I_{0}\left(f_{1}\right)=I_{0}(f)+f^{\prime}(0),
$$

where $f_{1}(x)=f(x+1)$.

By using iterative method, we get

$$
I_{0}\left(f_{n}\right)=I_{0}(f)+\sum_{i=0}^{n-1} f^{\prime}(i)
$$

where $f_{n}(x)=f(x+n)(n \in \mathbb{N})$.

As is well known, the fermionic $p$-adic integral on $\mathbb{Z}_{p}$ is defined by Kim to be

$$
\begin{aligned}
I_{-1}(f) & =\int_{\mathbb{Z}_{p}} f(x) d \mu_{-1}(x)=\lim _{N \rightarrow \infty} \sum_{x=0}^{p^{N}-1} f(x) \mu_{-1}\left(x+p^{N} \mathbb{Z}_{p}\right) \\
& =\lim _{N \rightarrow \infty} \sum_{x=0}^{p^{N}-1} f(x)(-1)^{x} \quad(\text { see }[26,27]) .
\end{aligned}
$$

From (5), we can derive

$$
I_{-1}\left(f_{n}\right)+(-1)^{n-1} I_{-1}(f)=2 \sum_{l=0}^{n-1}(-1)^{n-l-1} f(l)
$$

In particular, $n=1$, we have

$$
I_{-1}\left(f_{1}\right)+I_{-1}(f)=2 f(0) \quad(\text { see }[26,27])
$$

The purpose of this paper is to investigate several special polynomials related to Barnestype polynomials and give some identities including Witt's formula of their polynomials.

Finally, we give some identities of mixed-type Bernoulli and Euler polynomials.

\section{Barnes-type polynomials}

Let $a_{1}, a_{2}, \ldots, a_{r} \neq 0 \in \mathbb{C}_{p}$. Then, by (3), we get

$$
\begin{aligned}
\int_{\mathbb{Z}_{p}} & \cdots \int_{\mathbb{Z}_{p}} e^{\left(a_{1} x_{1}+a_{2} x_{2}+\cdots+a_{r} x_{r}+x\right) t} d \mu_{0}\left(x_{1}\right) \cdots d \mu_{0}\left(x_{r}\right) \\
= & \left(\prod_{i=1}^{r} a_{i}\right)\left(\frac{t^{r}}{\left(e^{a_{1} t}-1\right)\left(e^{a_{2} t}-1\right) \cdots\left(e^{a_{r} t}-1\right)}\right) e^{x t} \\
= & \left(\prod_{i=1}^{r} a_{i}\right) \sum_{n=0}^{\infty} B_{n}\left(x \mid a_{1}, a_{2}, \ldots, a_{r}\right) \frac{t^{n}}{n !} .
\end{aligned}
$$

From (8), we obtain the following Witt's formula for the Barnes-Bernoulli polynomials. 
Theorem 1 For $a_{1}, a_{2}, \ldots, a_{r} \neq 0 \in \mathbb{C}_{p}$, we have

$$
B_{n}\left(x \mid a_{1}, \ldots, a_{r}\right)=\left(\prod_{i=1}^{r} a_{i}\right)^{-1} \int_{\mathbb{Z}_{p}} \cdots \int_{\mathbb{Z}_{p}}\left(a_{1} x_{1}+\cdots+a_{r} x_{r}+x\right)^{n} d \mu_{0}\left(x_{1}\right) \cdots d \mu_{0}\left(x_{r}\right) .
$$

Note that

$$
\begin{aligned}
\left(a_{1} x_{1}+\cdots+a_{r} x_{r}\right)^{n} & =\sum_{l_{1}+\cdots+l_{r}=n}\left(\begin{array}{c}
n \\
l_{1}, \ldots, l_{r}
\end{array}\right) a_{1}^{l_{1}} x_{1}^{l_{1}} \cdots a_{r}^{l_{r}} x_{r}^{l_{r}} \\
& =\sum_{l_{1}+\cdots+l_{r}=n}\left(\begin{array}{c}
n \\
l_{1}, \ldots, l_{r}
\end{array}\right)\left(\prod_{i=1}^{r} a_{i}^{l_{i}}\right) x_{1}^{l_{1}} \cdots x_{r}^{l_{r}} .
\end{aligned}
$$

By (9) and Theorem 1, we obtain the following corollary.

Corollary 2 For $n \geq 2$, we have

$$
B_{n}\left(a_{1}, \ldots, a_{r}\right)=\sum_{l_{1}+\cdots+l_{r}=n}\left(\begin{array}{c}
n \\
l_{1}, \ldots, l_{r}
\end{array}\right)\left(\prod_{i=1}^{r} a_{i}^{l_{i}-1}\right) B_{l_{1}} \cdots B_{l_{r}}
$$

where $B_{n}=B_{n}(1)$ is the nth Bernoulli number.

From (2), we can easily derive the following integral equation:

$$
\int_{\mathbb{Z}_{p}} f(x) d \mu_{0}(x)=\frac{1}{d} \sum_{a=0}^{d-1} \int_{\mathbb{Z}_{p}} f(a+d x) d \mu_{0}(x),
$$

where $d \in \mathbb{N}$.

By (10), we get

$$
\begin{aligned}
\int_{\mathbb{Z}_{p}} \cdots \int_{\mathbb{Z}_{p}} e^{\left(a_{1} x_{1}+a_{2} x_{2}+\cdots+a_{r} x_{r}+x\right) t} d \mu_{0}(x) \\
=\frac{1}{d^{r}} \sum_{l_{1}=0}^{d-1} \cdots \sum_{l_{r}=0}^{d-1} \int_{\mathbb{Z}_{p}} \cdots \int_{\mathbb{Z}_{p}} e^{\left(l_{1} a_{1}+\cdots+l_{r} a_{r}+a_{1} d x_{1}+\cdots+a_{r} d x_{r}+x\right) t} d \mu_{0}\left(x_{1}\right) \cdots d \mu_{0}\left(x_{r}\right) \\
=\sum_{l_{1}=0}^{d-1} \cdots \sum_{l_{r}=0}^{d-1} \sum_{n=0}^{\infty} \frac{d^{n}}{d^{r}} \int_{\mathbb{Z}_{p}} \cdots \int_{\mathbb{Z}_{p}}\left(\frac{l_{1} a_{1}+\cdots+l_{r} a_{r}}{d}\right. \\
\left.\quad+a_{1} x_{1}+\cdots+a_{r} x_{r}+\frac{x}{d}\right)^{n} d \mu_{0}\left(x_{1}\right) \cdots d \mu_{0}\left(x_{r}\right) \frac{t^{n}}{n !} .
\end{aligned}
$$

By Theorem 1 and (11), we get

$$
B_{n}\left(x \mid a_{1}, \ldots, a_{r}\right)=d^{n-r} \sum_{l_{1}=0}^{d-1} \cdots \sum_{l_{r}=0}^{d-1} B_{n}\left(\frac{l_{1} a_{1}+\cdots+l_{r} a_{r}+x}{d} \mid a_{1}, \ldots, a_{r}\right) .
$$

Therefore, by (12), we obtain the following distribution relation for a Barnes-type Bernoulli polynomial. 
Theorem 3 For $n \geq 0$, we have

$$
B_{n}\left(x \mid a_{1}, \ldots, a_{r}\right)=d^{n-r} \sum_{l_{1}=0}^{d-1} \cdots \sum_{l_{r}=0}^{d-1} B_{n}\left(\frac{l_{1} a_{1}+\cdots+l_{r} a_{r}+x}{d} \mid a_{1}, \ldots, a_{r}\right) .
$$

From (4), we note that

$$
\int_{\mathbb{Z}_{p}} e^{a_{1}\left(x_{1}+n\right) t} d \mu_{0}\left(x_{1}\right)-\int_{\mathbb{Z}_{p}} e^{a_{1} x_{1} t} d \mu_{0}\left(x_{1}\right)=a_{1} t \sum_{l=0}^{n-1} e^{a_{1} l t}
$$

By (13), we get

$$
\int_{\mathbb{Z}_{p}} e^{a_{1} x_{1} t} d \mu_{0}\left(x_{1}\right)=\frac{a_{1} t}{e^{a_{1} n t}-1} \sum_{l=0}^{n-1} e^{a_{1} l t}
$$

From (14), we can derive

$$
\begin{aligned}
\int_{\mathbb{Z}_{p}} & \cdots \int_{\mathbb{Z}_{p}} e^{\left(a_{1} x_{1}+\cdots+a_{r} x_{r}\right) t} d \mu_{0}\left(x_{1}\right) \cdots d \mu_{0}\left(x_{r}\right) \\
= & \left(\frac{a_{1} t}{e^{n a_{1} t}-1}\right) \cdots\left(\frac{a_{r} t}{e^{n a_{r} t}-1}\right) \sum_{l_{1}, \ldots, l_{r}=0}^{n-1} e^{\left(a_{1} l_{1}+\cdots+a_{r} l_{r}\right) t} \\
= & \left(\prod_{i=1}^{r} a_{i}\right) \sum_{l_{1}, \ldots, l_{r}=0}^{n-1}\left(\sum_{k=0}^{\infty} B_{k}\left(n a_{1}, \ldots, n a_{r}\right) \frac{t^{k}}{k !}\right) \sum_{j=0}^{\infty}\left(a_{1} l_{1}+\cdots+a_{r} l_{r}\right)^{j} \frac{t^{j}}{j !} \\
= & \left(\prod_{i=1}^{r} a_{i}\right) \sum_{m=0}^{\infty}\left\{\sum_{l_{1}, \ldots, l_{r}=0}^{n-1} \sum_{j=0}^{m}\left(a_{1} l_{1}+\cdots+a_{r} l_{r}\right)^{j} B_{m-j}\left(n a_{1}, \ldots, n a_{r}\right)\left(\begin{array}{c}
m \\
j
\end{array}\right)\right\} \frac{t^{m}}{m !}
\end{aligned}
$$

By (15), we get

$$
\begin{aligned}
\int_{\mathbb{Z}_{p}} & \ldots \int_{\mathbb{Z}_{p}}\left(a_{1} x_{1}+\cdots+a_{r} x_{r}\right)^{m} d \mu_{0}\left(x_{1}\right) \cdots d \mu_{0}\left(x_{r}\right) \\
= & \left(\prod_{i=1}^{r} a_{i}\right) \sum_{l_{1}, \ldots, l_{r}=0}^{n-1} \sum_{j=0}^{m}\left(a_{1} l_{1}+\cdots+a_{r} l_{r}\right)^{j} B_{m-j}\left(n a_{1}, \ldots, n a_{r}\right)\left(\begin{array}{c}
m \\
j
\end{array}\right),
\end{aligned}
$$

where $n \in \mathbb{N}$ and $m \in \mathbb{Z} \geq 0$.

Therefore, by Theorem 1 and (16), we obtain the following theorem.

Theorem 4 For $n \in \mathbb{N}$ and $m \in \mathbb{Z}$ with $m \geq 0$, we have

$$
B_{m}\left(a_{1}, \ldots, a_{r}\right)=\sum_{l_{1}, \ldots, l_{r}=0}^{n-1} \sum_{j=0}^{m}\left(a_{1} l_{1}+\cdots+a_{r} l_{r}\right)^{j} B_{m-j}\left(n a_{1}, \ldots, n a_{r}\right)\left(\begin{array}{c}
m \\
j
\end{array}\right) .
$$

Moreover,

$$
B_{m}\left(x \mid a_{1}, \ldots, a_{r}\right)=\sum_{l_{1}, \ldots, l_{r}=0}^{n-1} \sum_{j=0}^{m}\left(a_{1} l_{1}+\cdots+a_{r} l_{r}+x\right)^{j} B_{m-j}\left(n a_{1}, \ldots, n a_{r}\right)\left(\begin{array}{c}
m \\
j
\end{array}\right)
$$


From (15), we observe that

$$
\begin{aligned}
\int_{\mathbb{Z}_{p}} & \cdots \int_{\mathbb{Z}_{p}} e^{\left(a_{1} x_{1}+\cdots+a_{r} x_{r}\right) t} d \mu_{0}\left(x_{1}\right) \cdots d \mu_{0}\left(x_{r}\right) \\
= & \left(\frac{a_{1} t}{e^{n a_{1} t}-1}\right) \cdots\left(\frac{a_{r} t}{e^{n a_{r} t}-1}\right) \sum_{l_{1}, \ldots, l_{r}=0}^{n-1} e^{\left(a_{1} l_{1}+\cdots+a_{r} l_{r}\right) t} \\
= & \sum_{l_{1}, \ldots, l_{r}=0}^{n-1} \frac{a_{1} t \cdots a_{r} t}{\left(e^{n a_{1} t}-1\right) \cdots\left(e^{n a_{r} t}-1\right)} e^{\left(\frac{a_{1} l_{1}+\cdots+a_{r} l_{r}}{n}\right) n t} \\
= & \left(\prod_{i=1}^{r} a_{i}\right) \sum_{l_{1}, \ldots, l_{r}=0}^{n-1} \sum_{m=0}^{\infty} B_{m}\left(\frac{a_{1} l_{1}+\cdots+a_{r} l_{r}}{n} \mid a_{1}, \ldots, a_{r}\right) n^{m} \frac{t^{m}}{m !} \\
= & \sum_{m=0}^{\infty}\left(\prod_{i=1}^{r} a_{i}\right) n^{m} \sum_{l_{1}, \ldots, l_{r}=0}^{n-1} B_{m}\left(\frac{a_{1} l_{1}+\cdots+a_{r} l_{r}}{n} \mid a_{1}, \ldots, a_{r}\right) \frac{t^{m}}{m !} .
\end{aligned}
$$

Thus, by (17), we get

$$
\begin{aligned}
\int_{\mathbb{Z}_{p}} & \cdots \int_{\mathbb{Z}_{p}}\left(a_{1} x_{1}+\cdots+a_{r} x_{r}\right)^{m} d \mu_{0}\left(x_{1}\right) \cdots d \mu_{0}\left(x_{r}\right) \\
= & \left(\prod_{i=1}^{r} a_{i}\right) n^{m} \sum_{l_{1}, \ldots, l_{r}=0}^{n-1} B_{m}\left(\frac{a_{1} l_{1}+\cdots+a_{r} l_{r}}{n} \mid a_{1}, \ldots, a_{r}\right),
\end{aligned}
$$

where $n \in \mathbb{N}$ and $m \in \mathbb{Z} \geq 0$.

Therefore, by Theorem 1 and (17), we obtain the following theorem.

Theorem 5 For $n \in \mathbb{N}$ and $m \geq 0$, we have

$$
B_{m}\left(a_{1}, \ldots, a_{r}\right)=n^{m} \sum_{l_{1}, \ldots, l_{r}=0}^{n-1} B_{m}\left(\frac{a_{1} l_{1}+\cdots+a_{r} l_{r}}{n} \mid a_{1}, \ldots, a_{r}\right)
$$

Moreover,

$$
B_{m}\left(x \mid a_{1}, \ldots, a_{r}\right)=n^{m} \sum_{l_{1}, \ldots, l_{r}=0}^{n-1} B_{m}\left(\frac{a_{1} l_{1}+\cdots+a_{r} l_{r}+x}{n} \mid a_{1}, \ldots, a_{r}\right) .
$$

Remark Let $a_{1}=1$ and $r=1$. Then we have

$$
\int_{\mathbb{Z}_{p}} e^{(x+n) t} d \mu_{0}(x)-\int_{\mathbb{Z}_{p}} e^{x t} d \mu_{0}(x)=t \sum_{l=0}^{n-1} e^{l t}
$$

Thus, we have

$$
\sum_{m=0}^{\infty}\left\{\int_{\mathbb{Z}_{p}}(x+n)^{m} d \mu_{0}(x)-\int_{\mathbb{Z}_{p}} x^{m} d \mu_{0}(x)\right\} \frac{t^{m}}{m !}(x)=t \sum_{l=0}^{n-1} \sum_{m=0}^{\infty} l^{m} \frac{t^{m}}{m !}
$$


By (19), we get

$$
\frac{1}{m+1}\left\{\int_{\mathbb{Z}_{p}}(x+n)^{m+1} d \mu_{0}(x)-\int_{\mathbb{Z}_{p}} x^{m+1} d \mu_{0}(x)\right\}=\sum_{l=0}^{n-1} l^{m}
$$

where $n \in \mathbb{N}$ and $m \in \mathbb{Z} \geq 0$.

It is easy to show that

$$
\int_{\mathbb{Z}_{p}} e^{(x+y) t} d \mu_{0}(y)=\frac{t}{e^{t}-1} e^{x t}=\sum_{n=0}^{\infty} B_{n}(x) \frac{t^{n}}{n !},
$$

where $B_{n}(x)$ is the $n$th Bernoulli polynomial.

Thus, by (21), we get

$$
\int_{\mathbb{Z}_{p}}(x+y)^{n} d \mu_{0}(y)=B_{n}(x) \quad(n \geq 0) .
$$

From (20) and (21), we note that

$$
\frac{1}{m+1}\left\{B_{m+1}(n)-B_{m+1}\right\}=\sum_{l=0}^{n-1} l^{m}
$$

where $m \in \mathbb{Z} \geq 0$ and $n \in \mathbb{N}$.

From (5) and (6), we can derive the following equation:

$$
\int_{\mathbb{Z}_{p}} e^{(x+1) t} d \mu_{-1}(x)+\int_{\mathbb{Z}_{p}} e^{x t} d \mu_{-1}(x)=2 .
$$

Thus, we have

$$
\int_{\mathbb{Z}_{p}} e^{(x+y) t} d \mu_{-1}(y)=\frac{2}{e^{t}+1} e^{x t}=\sum_{n=0}^{\infty} E_{n}(x) \frac{t^{n}}{n !},
$$

where $E_{n}(x)$ is the $n$th Euler polynomial.

Witt's formula for the Euler polynomials is given by

$$
\int_{\mathbb{Z}_{p}}(x+y)^{n} d \mu_{-1}(y)=E_{n}(x) \quad(n \geq 0)(\text { see }[26,27]) .
$$

When $x=0, E_{n}=E_{n}(0)$ are called the Euler numbers.

For $r \in \mathbb{N}$, the generating function of higher-order Euler polynomials can be derived from the multivariate $p$-adic fermionic integral on $\mathbb{Z}_{p}$ as follows:

$$
\begin{aligned}
\int_{\mathbb{Z}_{p}} \cdots \int_{\mathbb{Z}_{p}} e^{\left(x_{1}+\cdots+x_{r}+x\right) t} d \mu_{-1}\left(x_{1}\right) \cdots d \mu_{-1}\left(x_{r}\right) & =\left(\frac{2}{e^{t}+1}\right)^{r} e^{x t} \\
& =\sum_{n=0}^{\infty} E_{n}^{(r)}(x) \frac{t^{n}}{n !}
\end{aligned}
$$


Thus we get

$$
\int_{\mathbb{Z}_{p}} \cdots \int_{\mathbb{Z}_{p}}\left(x_{1}+\cdots+x_{r}+x\right)^{n} d \mu_{-1}\left(x_{1}\right) \cdots d \mu_{-1}\left(x_{r}\right)=E_{n}^{(r)}(x) \quad(n \in \mathbb{Z} \geq 0) .
$$

It is easy to show that

$$
\begin{aligned}
\int_{\mathbb{Z}_{p}} & \cdots \int_{\mathbb{Z}_{p}}\left(x_{1}+\cdots+x_{r}+x\right)^{n} d \mu_{-1}\left(x_{1}\right) \cdots d \mu_{-1}\left(x_{r}\right) \\
= & \sum_{l_{1}+\cdots+l_{r}=n}\left(\begin{array}{c}
n \\
l_{1}, \ldots, l_{r}
\end{array}\right) \int_{\mathbb{Z}_{p}} x_{1}^{l_{1}} d \mu_{-1}\left(x_{1}\right) \cdots \int_{\mathbb{Z}_{p}} x_{r-1}^{l_{r-1}} d \mu_{-1}\left(x_{r-1}\right) \\
& \times \int_{\mathbb{Z}_{p}}\left(x_{r}+x\right)^{l_{r}} d \mu_{-1}\left(x_{r}\right) \\
= & \sum_{l_{1}+\cdots+l_{r}=n}\left(\begin{array}{c}
n \\
l_{1}, \ldots, l_{r}
\end{array}\right) E_{l_{1}} E_{l_{2}} \cdots E_{l_{r-1}} E_{l_{r}}(x) .
\end{aligned}
$$

From (24) and (25), we have

$$
E_{n}^{(r)}(x)=\sum_{l_{1}+\cdots+l_{r}=n}\left(\begin{array}{c}
n \\
l_{1}, \ldots, l_{r}
\end{array}\right) E_{l_{1}} \cdots E_{l_{r-1}} E_{l_{r}}(x)
$$

When $x=0, E_{n}^{(r)}=E_{n}^{(r)}(0)$ are called the higher-order Euler numbers.

From (6), we note that

$$
\int_{\mathbb{Z}_{p}} e^{(x+n) t} d \mu_{-1}(x)+(-1)^{n-1} \int_{\mathbb{Z}_{p}} e^{x t} d \mu_{-1}(x)=2 \sum_{l=0}^{n-1}(-1)^{n-1-l} e^{l t} \quad(n \in \mathbb{N})
$$

Thus, by (27), we get

$$
\int_{\mathbb{Z}_{p}} e^{x t} d \mu_{-1}(x)=\frac{2}{e^{n t}+(-1)^{n-1}} \sum_{l=0}^{n-1}(-1)^{n-1-l} e^{l t}
$$

and

$$
\begin{aligned}
& \sum_{m=0}^{\infty}\left\{\int_{\mathbb{Z}_{p}}(x+n)^{m} d \mu_{-1}(x)+(-1)^{n-1} \int_{\mathbb{Z}_{p}} x^{m} d \mu_{-1}(x)\right\} \frac{t^{m}}{m !} \\
& =\sum_{m=0}^{\infty}\left\{2 \sum_{l=0}^{n-1}(-1)^{n-1-l} l^{m}\right\} \frac{t^{m}}{m !} \quad(n \in \mathbb{N}) .
\end{aligned}
$$

By comparing the coefficients on the both sides of (29), we get

$$
\int_{\mathbb{Z}_{p}}(x+n)^{m} d \mu_{-1}(x)+(-1)^{n-1} \int_{\mathbb{Z}_{p}} x^{m} d \mu_{-1}(x)=2 \sum_{l=0}^{n-1}(-1)^{n-1-l} l^{m}
$$

where $n \in \mathbb{N}$ and $m \in \mathbb{Z} \geq 0$.

Therefore, by (23) and (30), we obtain the following lemma. 
Lemma 6 For $m \geq 0, n \in \mathbb{N}$, we have

$$
E_{m}(n)+(-1)^{n-1} E_{m}=2 \sum_{l=0}^{n-1}(-1)^{n-1-l} l^{m} .
$$

Let us assume that $n \in \mathbb{N}$ with $n \equiv 1(\bmod 2)$.

Then, by (28), we get

$$
\int_{\mathbb{Z}_{p}} e^{x t} d \mu_{-1}(x)=\frac{2}{e^{n t}+1} \sum_{l=0}^{n-1}(-1)^{l} e^{l t} .
$$

Now, we consider the multivariate $p$-adic fermionic integral on $\mathbb{Z}_{p}$ related to the higherorder Euler numbers as follows:

$$
\begin{aligned}
\int_{\mathbb{Z}_{p}} & \cdots \int_{\mathbb{Z}_{p}} e^{\left(x_{1}+x_{2}+\cdots+x_{r}\right) t} d \mu_{-1}\left(x_{1}\right) \cdots d \mu_{-1}\left(x_{r}\right) \\
= & \left(\frac{2}{e^{n t}+1}\right)^{r} \sum_{l_{1}=0}^{n-1} \cdots \sum_{l_{r}=0}^{n-1}(-1)^{l_{1}+\cdots+l_{r}} e^{\left(l_{1}+\cdots+l_{r}\right) t} \\
= & \left(\sum_{l=0}^{\infty} E_{l}^{(r)} n^{l} \frac{t^{l}}{l !}\right) \sum_{l_{1}=0}^{n-1} \cdots \sum_{l_{r}=0}^{n-1}(-1)^{l_{1}+\cdots+l_{r}} \sum_{k=0}^{\infty}\left(l_{1}+\cdots+l_{r}\right)^{k} \frac{t^{k}}{k !} \\
= & \sum_{l_{1}=0}^{n-1} \cdots \sum_{l_{r}=0}^{n-1}(-1)^{l_{1}+\cdots+l_{r}} \sum_{m=0}^{\infty}\left\{\sum_{k=0}^{m}\left(\begin{array}{c}
m \\
k
\end{array}\right)\left(l_{1}+\cdots+l_{r}\right)^{k} n^{m-k} E_{m-k}^{(r)}\right\} \frac{t^{m}}{m !} \\
= & \sum_{m=0}^{\infty}\left\{\sum_{l_{1}=0}^{n-1} \cdots \sum_{l_{r}=0}^{n-1}(-1)^{l_{1}+\cdots+l_{r}} \sum_{k=0}^{m}\left(\begin{array}{c}
m \\
k
\end{array}\right) n^{m-k} E_{m-k}^{(r)}\left(l_{1}+\cdots+l_{r}\right)^{k}\right\} \frac{t^{m}}{m !} .
\end{aligned}
$$

Thus, from (32), we can derive the following equation:

$$
\begin{aligned}
\int_{\mathbb{Z}_{p}} & \cdots \int_{\mathbb{Z}_{p}}\left(x_{1} \cdots+x_{r}\right)^{m} d \mu_{-1}\left(x_{1}\right) \cdots d \mu_{-1}\left(x_{r}\right) \\
= & \sum_{l_{1}=0}^{n-1} \cdots \sum_{l_{r}=0}^{n-1} \sum_{k=0}^{m}(-1)^{l_{1}+\cdots+l_{r}}\left(\begin{array}{c}
m \\
k
\end{array}\right) E_{m-k}^{(r)} n^{m-k}\left(l_{1}+\cdots+l_{r}\right)^{k},
\end{aligned}
$$

where $m \geq 0$ and $n \in \mathbb{N}$ with $n \equiv 1(\bmod 2)$.

Therefore, by (24) and (33), we obtain the following theorem.

Theorem 7 For $m \geq 0$ and $n \in \mathbb{N}$ with $n \equiv 1(\bmod 2)$, we have

$$
E_{m}^{(r)}=\sum_{l_{1}=0}^{n-1} \cdots \sum_{l_{r}=0}^{n-1} \sum_{k=0}^{m}(-1)^{l_{1}+\cdots+l_{r}}\left(\begin{array}{c}
m \\
k
\end{array}\right) E_{m-k}^{(r)} n^{m-k}\left(l_{1}+\cdots+l_{r}\right)^{k},
$$

Moreover,

$$
E_{m}^{(r)}(x)=\sum_{l_{1}=0}^{n-1} \cdots \sum_{l_{r}=0}^{n-1} \sum_{k=0}^{m}(-1)^{l_{1}+\cdots+l_{r}}\left(\begin{array}{c}
m \\
k
\end{array}\right) E_{m-k}^{(r)} n^{m-k}\left(l_{1}+\cdots+l_{r}+x\right)^{k} .
$$


From (32), we note that

$$
\begin{aligned}
\int_{\mathbb{Z}_{p}} & \cdots \int_{\mathbb{Z}_{p}} e^{\left(x_{1}+\cdots+x_{r}\right) t} d \mu_{-1}\left(x_{1}\right) \cdots d \mu_{-1}\left(x_{r}\right) \\
= & \left(\frac{2}{e^{n t}+1}\right)^{r} \sum_{l_{1}=0}^{n-1} \cdots \sum_{l_{r}=0}^{n-1}(-1)^{l_{1}+\cdots+l_{r}} e^{\left(l_{1}+\cdots+l_{r}\right) t} \\
= & \sum_{l_{1}=0}^{n-1} \cdots \sum_{l_{r}=0}^{n-1}(-1)^{l_{1}+\cdots+l_{r}}\left(\frac{2}{e^{n t}+1}\right)^{r} e^{\left(\frac{l_{1}+\cdots+l_{r}}{n}\right) n t} \\
= & \sum_{m=0}^{\infty} \sum_{l_{1}=0}^{n-1} \cdots \sum_{l_{r}=0}^{n-1}(-1)^{l_{1}+\cdots+l_{r}} E_{m}^{(r)}\left(\frac{l_{1}+\cdots+l_{r}}{n}\right) n^{m} \frac{t^{m}}{m !}
\end{aligned}
$$

where $n \in \mathbb{N}$ with $n \equiv 1(\bmod 2)$.

Thus, by (34), we get

$$
\begin{array}{r}
\int_{\mathbb{Z}_{p}} \cdots \int_{\mathbb{Z}_{p}}\left(x_{1}+\cdots+x_{r}\right)^{m} d \mu_{-1}\left(x_{1}\right) \cdots d \mu_{-1}\left(x_{r}\right) \\
=\sum_{l_{1}=0}^{n-1} \cdots \sum_{l_{r}=0}^{n-1}(-1)^{l_{1}+\cdots+l_{r}} E_{m}^{(r)}\left(\frac{l_{1}+\cdots+l_{r}}{n}\right) n^{m}
\end{array}
$$

where $m \in \mathbb{Z} \geq 0, n \in \mathbb{N}$ with $n \equiv 1(\bmod 2)$.

Therefore by (24) and (35), we obtain the following theorem.

Theorem 8 For $m \in \mathbb{Z} \geq 0, n \in \mathbb{N}$ with $n \equiv 1(\bmod 2)$, we have

$$
E_{m}^{(r)}=n^{m} \sum_{l_{1}=0}^{n-1} \cdots \sum_{l_{r}=0}^{n-1}(-1)^{l_{1}+\cdots+l_{r}} E_{m}^{(r)}\left(\frac{l_{1}+\cdots+l_{r}}{n}\right)
$$

\section{Moreover,}

$$
E_{m}^{(r)}(x)=n^{m} \sum_{l_{1}=0}^{n-1} \cdots \sum_{l_{r}=0}^{n-1}(-1)^{l_{1}+\cdots+l_{r}} E_{m}^{(r)}\left(\frac{l_{1}+\cdots+l_{r}+x}{n}\right) .
$$

For $a_{1}, a_{2}, \ldots, a_{r} \in \mathbb{C}_{p} \backslash\{0\}$, let us consider the Barnes-type multiple Euler polynomials as follows:

$$
\begin{aligned}
\int_{\mathbb{Z}_{p}} & \cdots \int_{\mathbb{Z}_{p}} e^{\left(a_{1} x_{1}+\cdots+a_{r} x_{r}+x\right) t} d \mu_{-1}\left(x_{1}\right) \cdots d \mu_{-1}\left(x_{r}\right) \\
= & \left(\frac{2}{e^{a_{1} t}+1}\right) \times \cdots \times\left(\frac{2}{e^{a_{r} t}+1}\right) e^{x t} \\
= & \sum_{n=0}^{\infty} E_{n}\left(x \mid a_{1}, \ldots, a_{r}\right) \frac{t^{n}}{n !}
\end{aligned}
$$

When $x=0, E_{n}\left(a_{1}, \ldots, a_{r}\right)=E_{n}\left(0 \mid a_{1}, \ldots, a_{r}\right)$ is called the $n$th Barnes-type Euler number. 
For $d \in \mathbb{N}$ with $d \equiv 1(\bmod 2)$, we observe that

$$
\int_{\mathbb{Z}_{p}} f(x) d \mu_{-1}(x)=\sum_{a=0}^{d-1}(-1)^{a} \int_{\mathbb{Z}_{p}} f(a+d x) d \mu_{-1}(x)
$$

From (37), we can derive the following equation:

$$
\begin{aligned}
\int_{\mathbb{Z}_{p}} & \cdots \int_{\mathbb{Z}_{p}} e^{\left(a_{1} x_{1}+\cdots+a_{r} x_{r}\right) t} d \mu_{-1}\left(x_{1}\right) \cdots d \mu_{-1}\left(x_{r}\right) \\
= & \sum_{l_{1}=0}^{d-1} \cdots \sum_{l_{r}=0}^{d-1}(-1)^{l_{1}+\cdots+l_{r}} \int_{\mathbb{Z}_{p}} \cdots \int_{\mathbb{Z}_{p}} e^{\left\{a_{1} l_{1}+\cdots+a_{r} l_{r}+\left(a_{1} x_{1}+\cdots+a_{r} x_{r}\right) d\right\} t} d \mu_{-1}\left(x_{1}\right) \cdots d \mu_{-1}\left(x_{r}\right) \\
= & \sum_{n=0}^{\infty} d^{n} \sum_{l_{1}=0}^{d-1} \cdots \sum_{l_{r}=0}^{d-1}(-1)^{l_{1}+\cdots+l_{r}} \int_{\mathbb{Z}_{p}} \cdots \int_{\mathbb{Z}_{p}}\left(\frac{a_{1} l_{1}+\cdots+a_{r} l_{r}}{d}\right. \\
& \left.+a_{1} x_{1}+\cdots+a_{r} x_{r}\right)^{n} d \mu_{-1}\left(x_{1}\right) \cdots d \mu_{-1}\left(x_{r}\right) \frac{t^{n}}{n !}
\end{aligned}
$$

By (38), we get

$$
\begin{aligned}
& \int_{\mathbb{Z}_{p}} \cdots \int_{\mathbb{Z}_{p}}\left(a_{1} x_{1}+\cdots+a_{r} x_{r}\right)^{n} d \mu_{-1}\left(x_{1}\right) \cdots d \mu_{-1}\left(x_{r}\right) \\
& =d^{n} \sum_{l_{1}=0}^{d-1} \cdots \sum_{l_{r}=0}^{d-1}(-1)^{l_{1}+\cdots+l_{r}} \int_{\mathbb{Z}_{p}} \cdots \int_{\mathbb{Z}_{p}}\left(\frac{a_{1} l_{1}+\cdots+a_{r} l_{r}}{d}\right. \\
& \left.+a_{1} x_{1}+\cdots+a_{r} x_{r}\right)^{n} d \mu_{-1}\left(x_{1}\right) \cdots d \mu_{-1}\left(x_{r}\right) .
\end{aligned}
$$

Therefore, by (36) and (39), we obtain the following theorem.

Theorem 9 For $d \in \mathbb{N}$ with $d \equiv 1(\bmod 2), n \geq 0$, we have

$$
E_{n}\left(a_{1}, \ldots, a_{r}\right)=d^{n} \sum_{l_{1}=0}^{d-1} \cdots \sum_{l_{r}=0}^{d-1}(-1)^{l_{1}+\cdots+l_{r}} E_{n}\left(\frac{a_{1} l_{1}+\cdots+a_{r} l_{r}}{d} \mid a_{1}, \ldots, a_{r}\right) .
$$

Moreover,

$$
E_{n}\left(x \mid a_{1}, \ldots, a_{r}\right)=d^{n} \sum_{l_{1}=0}^{d-1} \ldots \sum_{l_{r}=0}^{d-1}(-1)^{l_{1}+\cdots+l_{r}} E_{n}\left(\frac{x+a_{1} l_{1}+\cdots+a_{r} l_{r}}{d} \mid a_{1}, \ldots, a_{r}\right)
$$

Remark Note that

$$
\begin{aligned}
E_{n}\left(x \mid a_{1}, \ldots, a_{r}\right) & =\sum_{l=0}^{n}\left(\begin{array}{l}
n \\
l
\end{array}\right) x^{l} E_{n-l}\left(a_{1}, \ldots, a_{r}\right) \\
& =\sum_{l=0}^{n}\left(\begin{array}{l}
n \\
l
\end{array}\right) x^{n-l} E_{l}\left(a_{1}, \ldots, a_{r}\right) .
\end{aligned}
$$


Thus, we have

$$
\begin{aligned}
\int_{\mathbb{Z}_{p}} & \cdots \int_{\mathbb{Z}_{p}} \cdots \int_{\mathbb{Z}_{p}}\left(a_{1} x_{1}+\cdots+a_{r} x_{r}\right. \\
& \left.+b_{1} y_{1}+\cdots+b_{s} y_{s}\right)^{n} d \mu_{-1}\left(y_{1}\right) \cdots d \mu_{-1}\left(y_{s}\right) d \mu_{0}\left(x_{1}\right) \cdots d \mu_{0}\left(x_{r}\right) \\
= & \int_{\mathbb{Z}_{p}} \cdots \int_{\mathbb{Z}_{p}} E_{n}\left(a_{1} x_{1}+\cdots+a_{r} x_{r} \mid b_{1}, \ldots, b_{s}\right) d \mu_{0}\left(x_{1}\right) \cdots d \mu_{0}\left(x_{r}\right) \\
= & \sum_{l=0}^{n}\left(\begin{array}{l}
n \\
l
\end{array}\right) E_{n-l}\left(b_{1}, \ldots, b_{s}\right) \int_{\mathbb{Z}_{p}} \cdots \int_{\mathbb{Z}_{p}}\left(a_{1} x_{1}+\cdots+a_{r} x_{r}\right)^{l} d \mu_{0}\left(x_{1}\right) \cdots d \mu_{0}\left(x_{r}\right) \\
= & \sum_{l=0}^{n}\left(\begin{array}{l}
n \\
l
\end{array}\right) E_{n-l}\left(b_{1}, \ldots, b_{s}\right) B_{l}\left(a_{1}, \ldots, a_{r}\right) .
\end{aligned}
$$

Now, we define mixed-type Barnes-type Euler and Bernoulli numbers as follows:

$$
\begin{aligned}
& E B_{n}\left(b_{1}, \ldots, b_{s} ; a_{1}, \ldots, a_{r}\right) \\
& =\int_{\mathbb{Z}_{p}} \cdots \int_{\mathbb{Z}_{p}} \cdots \int_{\mathbb{Z}_{p}}\left(a_{1} x_{1}+\cdots+a_{r} x_{r}\right. \\
& \left.\quad+b_{1} y_{1}+\cdots+b_{s} y_{s}\right)^{n} d \mu_{-1}\left(y_{1}\right) \cdots d \mu_{-1}\left(y_{s}\right) d \mu_{0}\left(x_{1}\right) \cdots d \mu_{0}\left(x_{r}\right),
\end{aligned}
$$

where $a_{1}, \ldots, a_{r}, b_{1}, \ldots, b_{s} \neq 0$.

By (40) and (41), we get

$$
E B_{n}\left(b_{1}, \ldots, b_{s} ; a_{1}, \ldots, a_{r}\right)=\sum_{l=0}^{n}\left(\begin{array}{l}
n \\
l
\end{array}\right) E_{n-l}\left(b_{1}, \ldots, b_{s}\right) B_{l}\left(a_{1}, \ldots, a_{r}\right) .
$$

\section{Competing interests}

The authors declare that they have no competing interests.

\section{Authors' contributions}

All authors contributed equally to this work. All authors read and approved the final manuscript.

\section{Acknowledgements}

Authors wish to express their sincere gratitude to the referees for their valuable suggestions and comments.

Received: 30 December 2014 Accepted: 21 January 2015 Published online: 11 February 2015

\section{References}

1. Agarwal, RP, Ryoo, CS: A numerical investigation on the structure of the roots of the twisted q-Bernoulli polynomials. Neural Parallel Sci. Comput. 14(1), 13-24 (2006)

2. Adamchik, VS: Contributions to the theory of the Barnes function. Int. J. Math. Comput. Sci. 9(1), 11-30 (2014)

3. Adamchik, VS: On the Barnes function. In: Proceedings of the 2001 International Symposium on Symbolic and Algebraic Computation, pp. 15-20. ACM, New York (2001) (electronic)

4. Bayad, A, Kim, T: Results on values of Barnes polynomials. Rocky Mt. J. Math. 43(6), 1857-1869 (2013)

5. Bayad, A, Kim, T, Kim, WJ, Lee, SH: Arithmetic properties of q-Barnes polynomials. J. Comput. Anal. Appl. 15(1), 111-117 (2013)

6. Billingham, J, King, AC: Uniform asymptotic expansions for the Barnes double gamma function. Proc. R. Soc. Lond. Ser. A 453(1964), 1817-1829 (1997)

7. Buschman, RG, Srivastava, HM: Convergence regions for some multiple Mellin-Barnes contour integrals representing generalized hypergeometric functions. Int. J. Math. Educ. Sci. Technol. 17(5), 605-609 (1986)

8. Carlitz, L: Some polynomials related to the Bernoulli and Euler polynomials. Util. Math. 19, 81-127 (1981)

9. Carlitz, L: Some remarks on the multiplication theorems for the Bernoulli and Euler polynomials. Glas. Mat. 16(36)(1) 3-23 (1981) 
10. Kim, DS, Kim, T, Komatsu, T, Lee, S-H: Barnes-type Daehee of the first kind and poly-Cauchy of the first kind mixed-type polynomials. Adv. Differ. Equ. 2014, 140 (2014)

11. Kim, DS, Kim, T: A study on the integral of the product of several Bernoulli polynomials. Rocky Mt. J. Math. 44(4), 1251-1263 (2014)

12. Kim, DS, Kim, T: q-Bernoulli polynomials and q-umbral calculus. Sci. China Math. 57(9), 1867-1874 (2014)

13. Kim, T: Barnes-type multiple $q$-zeta functions and $q$-Euler polynomials. J. Phys. A 43(25), 255201 (2010)

14. Kim, T: On Euler-Barnes multiple zeta functions. Russ. J. Math. Phys. 10(3), 261-267 (2003)

15. Kim, T, Rim, S-H: On Changhee-Barnes' $q$-Euler numbers and polynomials. Adv. Stud. Contemp. Math. (Kyungshang) 9(2), 81-86 (2004)

16. Kim, T: $p$-Adic $q$-integrals associated with the Changhee-Barnes' $q$-Bernoulli polynomials. Integral Transforms Spec. Funct. 15(5), 415-420 (2004)

17. Kim, T, Dolgy, DV, Kim, DS, Rim, S-H: A note on the identities of special polynomials. Ars Comb. 113A, 97-106 (2014)

18. Kim, T: On $p$-adic interpolating function for $q$-Euler numbers and its derivatives. J. Math. Anal. Appl. 339(1), 598-608 (2008)

19. Kim, T: On the analogs of Euler numbers and polynomials associated with $p$-adic $q$-integral on $\mathbb{Z}_{p}$ at $q=-1$. J. Math. Anal. Appl. 331(2), 779-792 (2007)

20. Kim, T: On p-adic q-I-functions and sums of powers. J. Math. Anal. Appl. 329(2), 1472-1481 (2007)

21. Kim, T: $q$-Volkenborn integration. Russ. J. Math. Phys. 9(3), 288-299 (2002)

22. Kim, T: $q$-Bernoulli numbers and polynomials associated with Gaussian binomial coefficients. Russ. J. Math. Phys. 15(1), 51-57 (2008)

23. Kim, T: Power series and asymptotic series associated with the $q$-analog of the two-variable $p$-adic $L$-function. Russ. J. Math. Phys. 12(2), 186-196 (2005)

24. Kim, T: Non-Archimedean $q$-integrals associated with multiple Changhee $q$-Bernoulli polynomials. Russ. J. Math. Phys. 10(1), 91-98 (2003)

25. Kim, T: Note on the Euler q-zeta functions. J. Number Theory 129(7), 1798-1804 (2009)

26. Kim, T: $q$-Euler numbers and polynomials associated with $p$-adic $q$-integrals. J. Nonlinear Math. Phys. 14(1), 15-27 (2007)

27. Kim, T: Symmetry $p$-adic invariant integral on $\mathbb{Z}_{p}$ for Bernoulli and Euler polynomials. J. Differ. Equ. Appl. 14(12), 1267-1277 (2008)

28. Liu, FB: Barnes integral representations of some special functions. Neimenggu Daxue Xuebao Ziran Kexue 26(4), 387-395 (1995)

29. Park, J-W: New approach to $q$-Bernoulli polynomials with weight or weak weight. Adv. Stud. Contemp. Math. (Kyungshang) 24(1), 39-44 (2014)

30. Ruijsenaars, SNM: On Barnes' multiple zeta and gamma functions. Adv. Math. 156(1), 107-132 (2000)

31. Schuster, R: A generalization of the Barnes G-function. Z. Anal. Anwend. 11(2), 229-236 (1992)

32. Seo, J-J, Rim, SH, Kim, T, Lee, SH: Sums products of generalized Daehee numbers. Proc. Jangjeon Math. Soc. 17(1), 1-9 (2014)

33. Srivastava, HM, Kim, T, Simsek, Y: q-Bernoulli numbers and polynomials associated with multiple q-zeta functions and basic L-series. Russ. J. Math. Phys. 12(2), 241-268 (2005)

\section{Submit your manuscript to a SpringerOpen ${ }^{\ominus}$ journal and benefit from:}

- Convenient online submission

Rigorous peer review

- Immediate publication on acceptance

- Open access: articles freely available online

- High visibility within the field

- Retaining the copyright to your article 